Article

\title{
Isoliquiritigenin Attenuates Atherogenesis in Apolipoprotein E-Deficient Mice
}

\author{
Fen Du ${ }^{1, \dagger}$, Quzhen Gesang ${ }^{1,2,+}$, Jia Cao ${ }^{1}$, Mei Qian ${ }^{1}$, Li Ma ${ }^{1}$, Dongfang $\mathrm{Wu}^{3}$ and Hong Yu ${ }^{1, *}$ \\ 1 Department of Biochemistry and Molecular Biology, Hubei Provincial Key Laboratory of Developmentally \\ Originated Disease, Wuhan University School of Basic Medical Sciences, 185 Donghu Road, Bldg. 2, 2-209, \\ Wuhan 430071, China; fen.du@whu.edu.cn (F.D.); gsqz123@163.com (Q.G.); caojia1210@whu.edu.cn (J.C.); \\ qianmei@whu.edu.cn (M.Q.); 2013203010022@whu.edu.cn (L.M.) \\ 2 Medical College, Tibet University, Lhasa 850000, China \\ 3 Department of Pharmacy, Zhongnan Hospital of Wuhan University, Wuhan 430071, China; \\ dfwu2010@whu.edu.cn \\ * Correspondence: yu.hong@whu.edu.cn; Tel.: +86-27-6875-9795 \\ + These authors contributed equally to this work.
}

Academic Editor: Shaker A. Mousa

Received: 28 August 2016; Accepted: 11 November 2016; Published: 18 November 2016

\begin{abstract}
Isoliquiritigenin (ISL) exhibits antioxidation and anti-inflammation activity. We sought to investigate the effects and mechanism of ISL on the development of atherosclerotic lesions in apolipoprotein E-deficient $\left(\mathrm{apoE}^{-/-}\right)$mice. Firstly, we determined that ISL reduced the mRNA levels of inflammatory factors interleukin 6 (IL-6), tumor necrosis factor $\alpha$ (TNF- $\alpha$ ), and monocyte chemotactic protein-1 (MCP-1), while it increased the expression of several lipoprotein-related genes in peritoneal macrophages treated with lipopolysaccharide (LPS). ISL also enhanced peroxisome proliferator-activated receptor gamma (PPAR $\gamma$ ) protein levels and reversed the changes of ATP-binding cassette transporter A (ABCA1) and cluster of differentiation 36 (CD36) in macrophages treated with oxidative low-density lipoprotein (ox-LDL). Then, in an in vivo study, female apoE ${ }^{-/-}$ mice were fed a Western diet with ISL $(0,20,100 \mathrm{mg} / \mathrm{kg} /$ day) added for 12 weeks. We found that ISL decreased the plasma cholesterol levels of very low-density lipoprotein (VLDL)/LDL, promoted plasma superoxide dismutase (SOD) and paraoxonase-1 (PON1) activities, and decreased plasma IL-6, TNF- $\alpha$, and MCP-1 levels. Moreover, ISL significantly reduced the atherosclerotic lesions and hepatic steatosis in apoE $\mathrm{E}^{-/-}$mice. In the liver, ISL altered the expression of several key genes (such as $S R B I, A B C A 1, A B C G 8, P P A R \gamma$, and FASN) involving cholesterol-selective uptake and excretion into bile, triglyceride (TG) biosynthesis, and inflammation. These results suggest that the atheroprotective effects of ISL are due to the improvement of lipid metabolism, antioxidation, and anti-inflammation, which involve PPAR $\gamma$-dependent signaling.
\end{abstract}

Keywords: isoliquiritigenin; atherosclerosis; cholesterol flux; anti-inflammation; anti-oxidation

\section{Introduction}

Atherosclerosis is characterized by the development of low-grade arterial inflammatory lesions, and is associated with oxidative stress and dyslipidemia [1-3]. Lipid accumulation and oxidation induces the release of inflammatory factors within the arterial wall and is responsible for atherosclerotic plaque development $[4,5]$. Oxidative stress reflects the excessive production of reactive oxygen species (ROS). The main sources of ROS in atherosclerotic vessels are endothelial cells, smooth muscle cells, and macrophages [6], and the presence of ROS leads to the oxidative modification of low-density lipoproteins (LDL). Macrophages aggressively take up oxidized lipids, and become foam cells that initiate the formation of atherosclerotic lesions. Macrophage foam cells release proinflammatory 
cytokines and thereby further exaggerate arterial wall inflammation [7]. To date, the available therapies for atherosclerosis simply alleviate hyperlipidemia. The mortality of atherosclerosis-related cardiovascular disease is still unacceptably high; therefore, new therapeutic approaches are needed for atherosclerosis. Drugs with more comprehensive therapeutic effects on elevated plasma lipids, lipoprotein oxidation, and vascular inflammation will have important clinical value for the treatment and prevention of atherosclerosis [8].

Isoliquiritigenin (ISL) is a bioactive ingredient of flavonoids extracted from licorice root [9]. ISL reportedly has numerous pharmacological properties-including anti-inflammatory, antimicrobial, antioxidative, and anticancer activities-and immunoregulatory, hepatoprotective, and cardioprotective effects [10,11]. ISL potently inhibits the activation of the NLRP3 inflammasome and improves diet-induced adipose tissue inflammation [12]. ISL decreases phosphorylated c-Jun N-terminal kinase (JNK) expression in macrophages induced by palmitic acid, and suppresses the induced inflammatory changes by inhibiting nuclear factor kappa $\mathrm{B}(\mathrm{NF} \kappa \mathrm{B})$ activation [13]. Wu et al. demonstrated that ISL inhibits interferon $\gamma$ induced inflammation in hepatocytes by influencing the activation of JAK1/STAT1, IRF3/MyD88, ERK/MAPK, JNK/MAPK, and PI3K/Akt signaling pathways [14]. ISL blocks the ROS generation induced by insulin in 3T3-L1 cells and suppresses lipid accumulation [15]. Cao et al. proved that ISL protects against oxidative stress in HepG2 cells by activating the nuclear factor erythroid 2-related factor (Nrf2) antioxidant response and increasing the expression of its target genes [16]. ISL was also shown to protect HT22 hippocampal neuronal cells from glutamate-induced oxidative stress and mitochondrial damage [17]. Kim et al. reported that ISL has the ability to protect hepatocytes from Western diet-induced oxidative injury to the liver and from liver $\mathrm{X}$ receptor $\alpha(\mathrm{LXR} \alpha)$-dependent hepatic steatosis via JNK1 inhibition [18].

On the basis of these features, we hypothesize that ISL may have antiatherosclerosis activities. We show herein that ISL can indeed reduce the development of atherosclerotic plaques in apolipoproteindeficient $\left(\mathrm{apoE}^{-/-}\right)$mice. We also confirm the antioxidative and anti-inflammatory effects of ISL in vitro and in vivo and further explore the mechanism of the lipid-regulation function of ISL in mice.

\section{Results}

\subsection{ISL Suppresses the Inflammatory Response of Macrophages to Lipopolysaccharide (LPS)}

To evaluate the cytotoxic effect of ISL, HeLa cells were treated with ISL for $48 \mathrm{~h}$, and cell viability was measured using the MTT assay. As shown in Figure S1a, a low concentration of ISL $(<1 \mu \mathrm{g} / \mathrm{mL})$ exhibited no obvious cytotoxicity after $48 \mathrm{~h}$ of treatment, whereas cell viability was obviously decreased with treatment using $>5 \mu \mathrm{g} / \mathrm{mL}$. Based on this observation, the low-dose $(<1 \mu \mathrm{g} / \mathrm{mL})$ ISL treatment was chosen for our experiments. We treated peritoneal macrophages with LPS at $50 \mathrm{ng} / \mathrm{mL}$ and ISL at varied concentrations. Cell viability was significantly decreased in LPS-treated cells and recovered in cells cotreated with a low concentration of ISL $(<1 \mu \mathrm{g} / \mathrm{mL}$; Figure S1b). These results showed that ISL, at low concentrations, could protect cells against LPS-induced inflammatory damage.

To further investigate the effects of ISL on the inflammatory response to LPS, we treated peritoneal macrophages with $50 \mathrm{ng} / \mathrm{mL}$ LPS and $0.1 \mu \mathrm{g} / \mathrm{mL}$ ISL for $6 \mathrm{~h}$. The expression of inflammatory factors were determined by quantitative polymerase chain reaction (qPCR) and showed that interleukin $6(I L-6)$, tumor necrosis factor $\alpha$ (TNF- $\alpha$ ), and monocyte chemotactic protein-1 (MCP-1) mRNA levels were significantly increased in LPS-treated macrophages, but reduced in cells treated with $0.1 \mu \mathrm{g} / \mathrm{mL}$ ISL (Figure 1a-c). These results verified that ISL effectively suppressed the LPS-induced inflammation in macrophages.

The ability of cells to maintain intracellular cholesterol homeostasis may be affected by the cellular inflammatory status. Therefore, we examined the effect of ISL on the expression of some cholesterol flux-related genes under LPS stimulation in macrophages. LXR $\alpha$, ATP-binding cassette transporter A1 ( $A B C A 1)$, and cluster of differentiation 36 (CD36) mRNA expression were all disturbed in macrophages treated with $50 \mathrm{ng} / \mathrm{mL}$ LPS, and were improved when the cells were cocultured with $0.1 \mu \mathrm{g} / \mathrm{mL}$ ISL 
(Figure $1 \mathrm{~d}-\mathrm{f}$ ). These results indicated that the anti-inflammatory activity of ISL might be correlated with the ability to upregulate the cholesterol flux-related genes in macrophages.

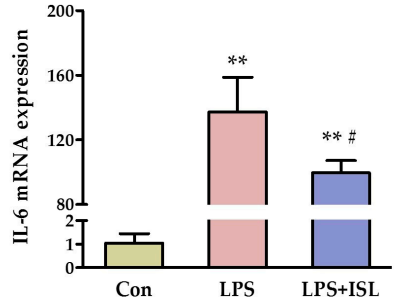

(a)

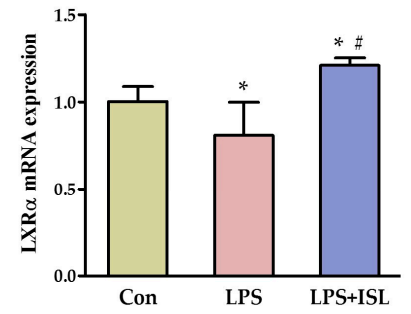

(d)

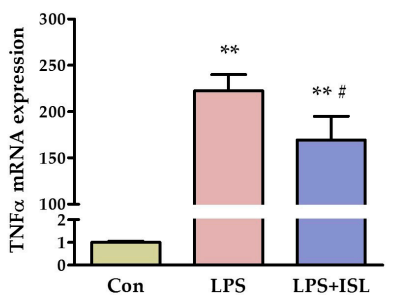

(b)

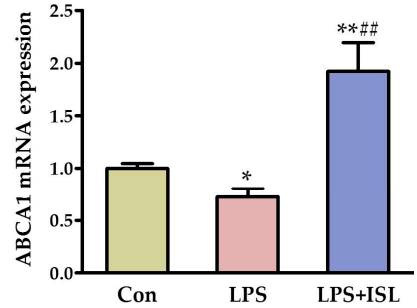

(e)

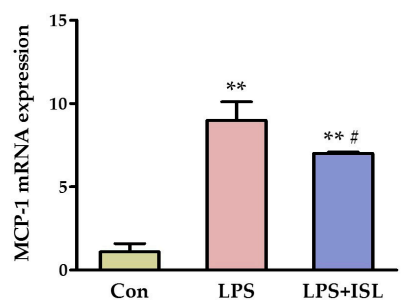

(c)

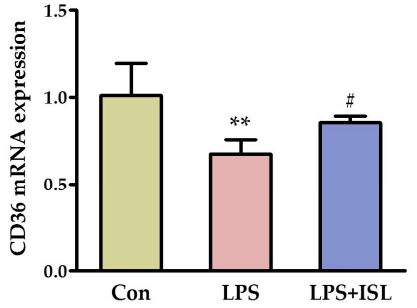

(f)

Figure 1. Isoliquiritigenin (ISL) reduced lipopolysaccharide (LPS)-induced inflammatory response and increased the expression of genes involved in cholesterol homeostasis in macrophages. The mRNA levels of inflammation-associated cytokines interleukin 6 (IL-6) (a); tumor necrosis factor $\alpha$ (TNF- $\alpha)(\mathbf{b})$; monocyte chemotactic protein-1 (MCP-1) (c); and lipid flux-related receptors liver $\mathrm{X}$ receptor $\alpha(L X R \alpha)(\mathbf{d})$; ATP-binding cassette transporter A1 (ABCA1) (e); and cluster of differentiation 36 (CD36) (f) were determined using qPCR. Peritoneal macrophages from apolipoprotein E-deficient $\left(\right.$ apoE $\left.^{-/-}\right)$mice were treated with LPS $(50 \mathrm{ng} / \mathrm{mL})$ and ISL $(0.1 \mu \mathrm{g} / \mathrm{mL})$ for $6 \mathrm{~h}$. Total RNA was extracted and reverse transcribed into cDNA $\left(n=3-4 ;{ }^{*} p<0.05,{ }^{* *} p<0.01\right.$ vs. control group, \# $p<0.05$, \#\# $p<0.01$ vs. LPS-induced group).

(a)

$$
\text { PPAR } \gamma
$$

$\beta$-actin

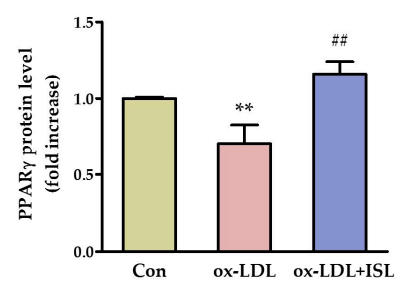

(b)
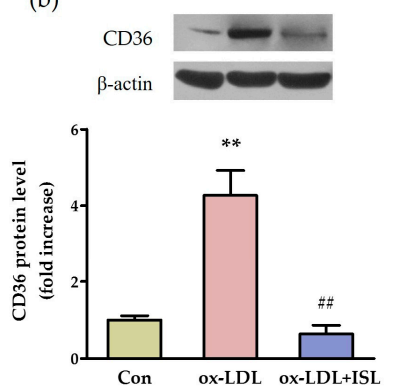

(c)
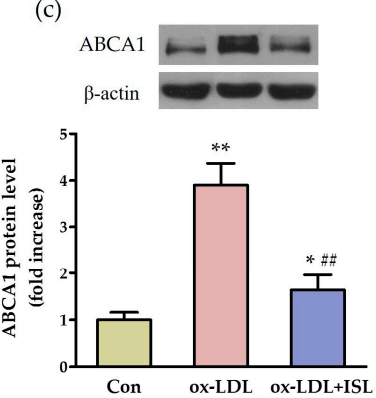

Figure 2. ISL affects expression of cholesterol-metabolism-related genes in macrophages induced by oxidative low-density lipoprotein (ox-LDL). The protein levels of peroxisome proliferator-activated receptor gamma $(\operatorname{PPAR} \gamma)(\mathbf{a})$; CD36 (b); and ABCA1 (c) were determined using Western blot analysis. Peritoneal macrophages from mice were coincubated with $50 \mu \mathrm{g} / \mathrm{mL}$ ox-LDL and $0.5 \mu \mathrm{g} / \mathrm{mL}$ ISL for $12 \mathrm{~h}$, then collected and lysed using radioimmunoprecipitation assay (RIPA) buffer. Appropriate amounts of proteins were separated using sodium dodecyl sulfate polyacrylamide gel electrophoresis (SDS-PAGE), and target proteins were detected as described in the Methods section. Relative fold increases of each protein are shown $\left(n=3-5 ;{ }^{*} p<0.05,{ }^{* *} p<0.01 \mathrm{vs.} \mathrm{control} \mathrm{group,}\right.$ $\#$ \# $p<0.01$ vs. ox-LDL-induced group).

\subsection{ISL Affects Cholesterol-Flux-Related Protein Expression in Macrophages Treated with Oxidative-LDL}

To investigate whether ISL can improve cholesterol flux in macrophages loaded with lipids, we treated peritoneal macrophages with $50 \mu \mathrm{g} / \mathrm{mL}$ oxidative-LDL (ox-LDL) and $0.5 \mu \mathrm{g} / \mathrm{mL}$ ISL for $12 \mathrm{~h}$. Western blot was performed to compare the protein levels of lipid metabolism-regulating-related nuclear 
receptor and cholesterol-flux receptors. The results showed that ox-LDL treatment significantly decreased the peroxisome proliferator-activated receptor gamma (PPAR $\gamma$ ) protein level in peritoneal macrophages, which was increased in ISL-treated cells (Figure 2a). CD36 and ABCA1 proteins, which mediate the ox-LDL influx and cholesterol efflux, were upregulated under ox-LDL treatment, whereas ISL significantly attenuated ox-LDL-induced CD36 and ABCA1 protein expression (Figure 2b,c). Collectively, these data indicated that ISL may modulate the expression of key genes in cholesterol flux through the upregulation of PPAR $\gamma$ in cells.

\subsection{ISL Ameliorates Plasma Lipid Levels and Inhibits Oxidative Stress and Inflammation in ApoE ${ }^{-/-}$Mice Fed a Western Diet}

To examine the effects of ISL on atherosclerosis, we fed $\mathrm{apoE}^{-/-}$mice a Western diet supplemented with ISL $(0,20$, or $100 \mathrm{mg} / \mathrm{kg})$ per day for 12-week. Fasting plasma lipid levels were monitored at 6-week intervals, then the mice were euthanized (endpoint) for atherosclerosis analysis. The results showed that plasma total cholesterol (TC) and triglyceride (TG) levels were dramatically increased on the Western diet. The TC level was reduced in mice fed with ISL, but the TG level was not altered (Figure 3a,b). Lipoprotein profiles were compared using fast protein liquid chromatography (FPLC), which showed that ISL significantly reduced the cholesterol level in very low-density lipoprotein (VLDL) and LDL fractions, but did not affect the high-density lipoprotein (HDL) fraction (Figure 3c). On the other hand, administration of ISL did not cause adverse effects in vivo, and no alterations were observed in body weight or spleen weight in the mice (Figure S2a,b).

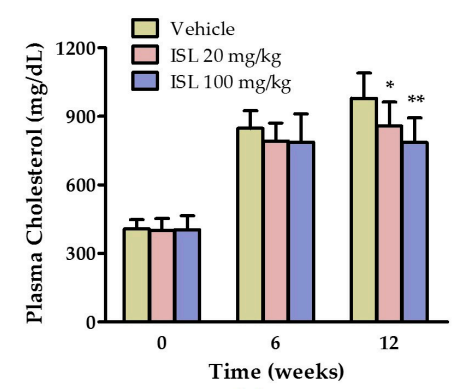

(a)

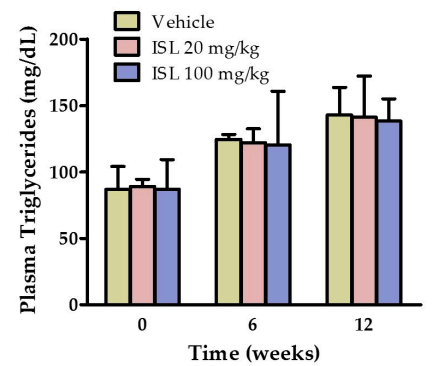

(b)

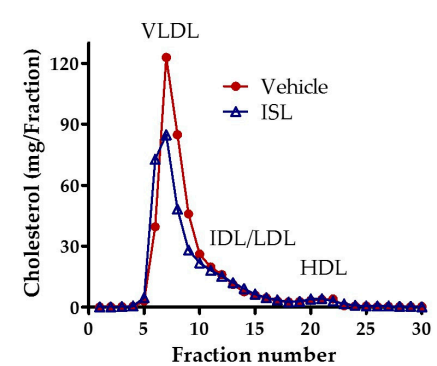

(c)
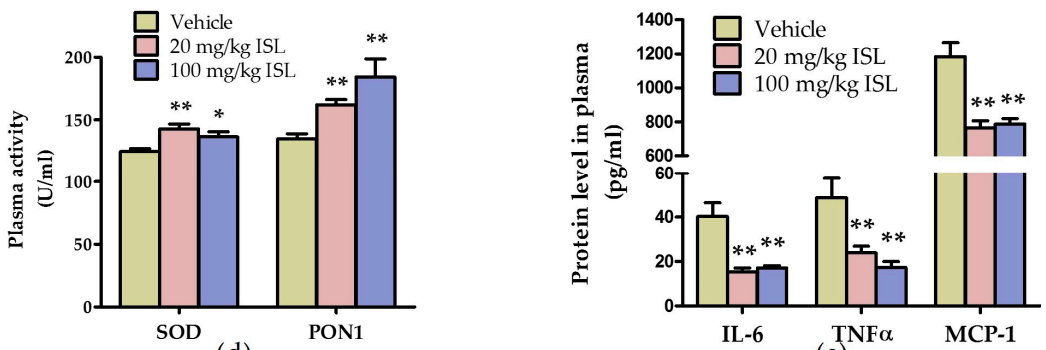

$(\mathrm{e})^{\mathrm{TN}}$

Figure 3. The effects of ISL on plasma lipids levels and antioxidative and anti-inflammatory status in $\mathrm{apoE}^{-/-}$mice fed a Western diet. Twenty-week old female apoE $\mathrm{E}^{-/-}$mice were fed an AIN76A Western diet supplemented with $0.5 \%$ sodium carboxymethyl cellulose (CMC-Na) or ISL $(20 \mathrm{mg} / \mathrm{kg} / \mathrm{day}$ or $100 \mathrm{mg} / \mathrm{kg} /$ day) for 12-week, and then sacrificed. The plasma total cholesterol (a) and triglyceride (b) levels were measured at the indicated time as described in the Methods section $\left(n=8-10 ;{ }^{*} p<0.05\right.$, ** $p<0.01$ vs. vehicle group at the same time point); (c) plasma lipoprotein profiles in apoE $\mathrm{E}^{-/-}$mice with or without high-dose ISL (100 mg/kg/day) supplementation. Lipoproteins in pooled plasma were size-fractionated by fast protein liquid chromatography (FPLC), and the cholesterol contents within lipoprotein fractions (very low-density lipoprotein (VLDL), LDL, and high-density lipoprotein (HDL)) were measured; (d) the plasma superoxide dismutase (SOD) and paraoxonase-1 (PON1) activities of apoE $\mathrm{E}^{-/-}$mice were measured at the endpoint using an enzymatic colorimetric assay $(n=8-10$; ${ }^{*} p<0.05,{ }^{* *} p<0.01$ vs. vehicle group); (e) plasma IL-6, TNF- $\alpha$, and MCP-1 levels were determined using enzyme-linked immunosorbent assay (ELISA) $\left(n=5-7 ;{ }^{* *} p<0.01\right.$ vs. vehicle group). 
The activities of antioxidative enzymes, including superoxide dismutase (SOD) and HDL-associated paraoxonase-1 (PON1), reflect the plasma redox status in mice. As shown in Figure 3d, the plasma SOD and PON1 activities were significantly increased in $\mathrm{apoE}^{-/-}$mice fed with ISL. We also determined the concentrations of plasma inflammatory cytokines in $\mathrm{apoE}^{-/-}$mice. ISL effectively reduced plasma IL-6, TNF- $\alpha$, and MCP-1 levels (Figure 3e). Taken together, these data indicated that ISL improved the plasma antioxidative and anti-inflammatory status in $\mathrm{apoE}^{-/-}$mice.

\subsection{ISL Attenuates Atherosclerosis in $A p o E^{-/-}$Mice}

The impact of ISL on atherosclerotic lesion development in $\mathrm{apoE}^{-/-}$mice was determined by visualizing the lesions on aortic root cross-sections and en face aortas with neutral oil red $\mathrm{O}$ staining and quantitative analysis (Figure 4). In the aortic roots, the mean lesion area of $\mathrm{apoE}^{-/-}$mice fed with ISL was notably decreased compared to the vehicle group, but there was no significant difference between two groups fed with different dosages of ISL (vehicle: $0.504 \pm 0.056 \mathrm{~mm}^{2}$; low-dose ISL: $0.445 \pm 0.043 \mathrm{~mm}^{2}$; high-dose ISL: $0.438 \pm 0.039 \mathrm{~mm}^{2}$; Figure $\left.4 \mathrm{a}, \mathrm{b}\right)$. The mean lesion areas were reduced by $16 \%$ and $19 \%$ in the low-dose and high-dose ISL groups, respectively. The percentage of lesion area in the en face aorta was also analyzed, and both doses of ISL significantly reduced atherosclerotic lesions in the aortic arch and total aorta compared with the vehicle group (Figure 4c-e). These data suggested that ISL reduced the development of atherosclerosis in apoE ${ }^{-/-}$mice.

(a)
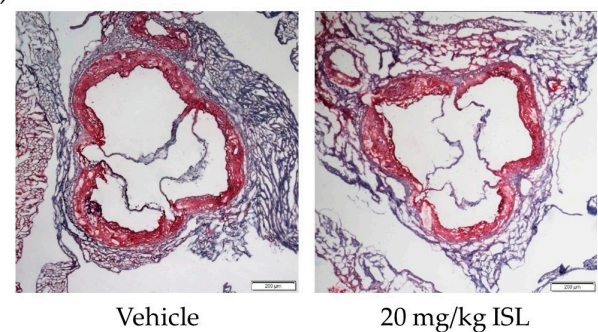

$20 \mathrm{mg} / \mathrm{kg}$ ISL

(c)

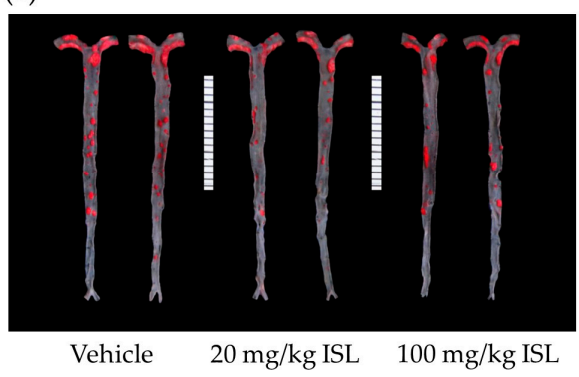

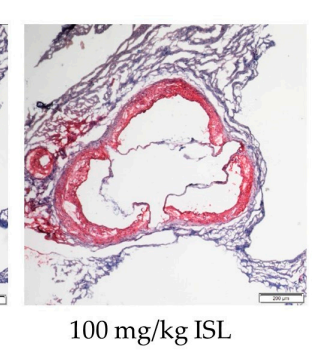

(d)

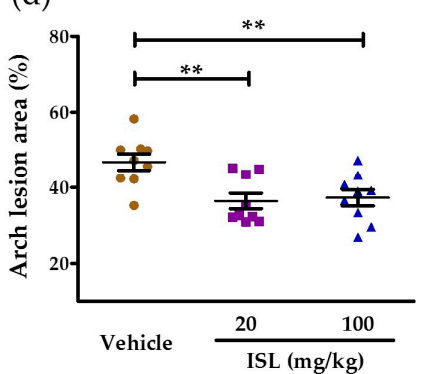

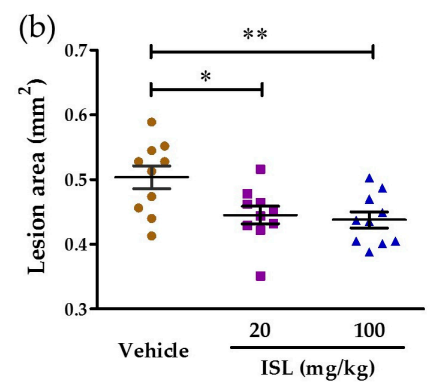

(e)

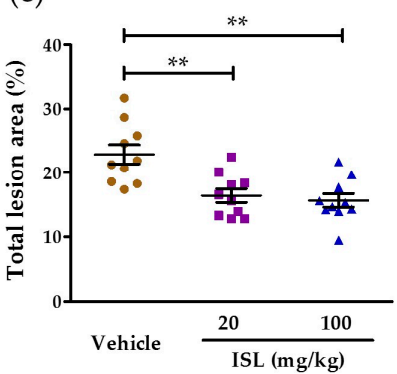

Figure 4. Atherosclerotic lesion areas in the aortic root and aorta en face of apoE $\mathrm{E}^{-/-}$mice. (a) Representative atherosclerotic lesions of the aortas examined using oil red O-stained cross-sections of the aortic root $(8 \mu \mathrm{m}$ serial sections, $40 \times)$; (b) quantitative analysis of the cross-sectional lesion areas of the aortic roots; (c) representative en face aortas' images; $(\mathbf{d})$ the percentages of lesion area in the aortic arch; (e) total lesion areas analyzed using the Mann-Whitney test $\left(n=9-10 ;{ }^{*} p<0.05,{ }^{* *} p<0.01\right.$ vs. vehicle group).

\subsection{ISL Reduces Hepatic Steatosis and Alters Hepatic Gene Expression in ApoE ${ }^{-/-}$Mice}

To examine the effects of ISL on hepatic steatosis in apoE $\mathrm{E}^{-/-}$mice, we performed hematoxylin and eosin (HE) staining and oil red $\mathrm{O}$ staining in liver slides. Lipid accumulation was significantly attenuated in mice fed with both doses of ISL (Figure 5a-c). Meanwhile, lipids in mouse liver were extracted and analyzed, hepatic TC, free cholesterol (FC), and TG levels were significantly decreased in $a \mathrm{poE}^{-/-}$mice fed with ISL compared with the vehicle group (Figure 5d). 
(a)

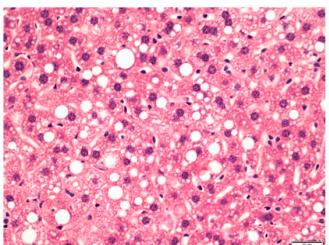

Vehicle $(400 \times)$

(b)

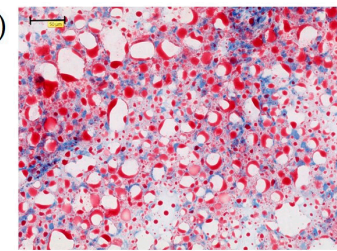

Vehicle $(200 \times)$

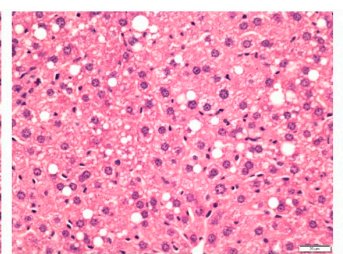

$20 \mathrm{mg} / \mathrm{kg}$ ISL $(400 \times)$

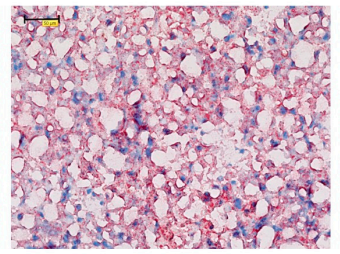

$20 \mathrm{mg} / \mathrm{kg}$ ISL $(200 \times)$

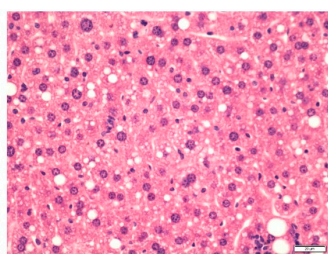

$100 \mathrm{mg} / \mathrm{kg}$ ISL $(400 \times)$

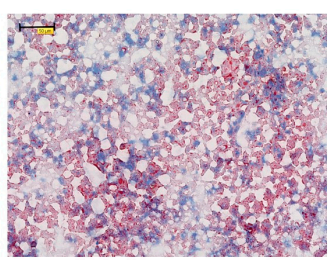

$100 \mathrm{mg} / \mathrm{kg}$ ISL $(200 \times)$ (c)

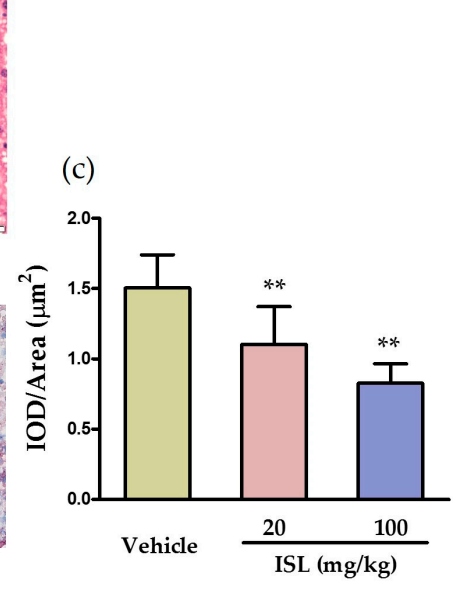

(d)

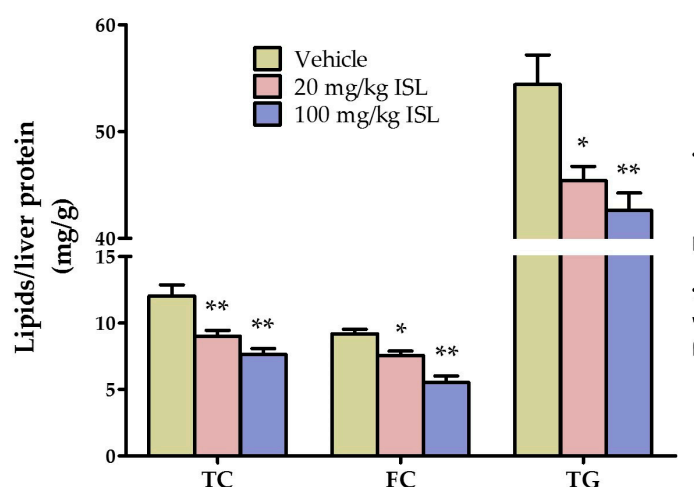

(e)

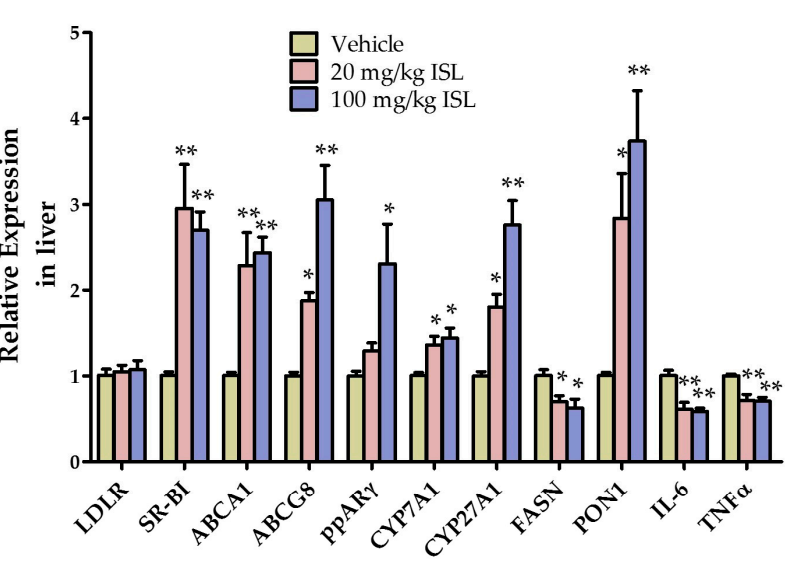

Figure 5. ISL attenuates hepatic steatosis by altering the expression of lipoprotein-metabolism-related genes. (a) Histochemical analysis of paraformaldehyde-fixed liver tissue was performed using hematoxylin and eosin (HE) staining (5 $\mu \mathrm{m}$ serial sections, 400×); (b) histochemical analysis of fresh liver tissue was performed by oil red O staining ( $5 \mu \mathrm{m}$ serial sections, $200 \times)$; (c) quantitative analysis of the lipid content in liver; (d) the amounts of total cholesterol (TC), free cholesterol (FC), and triglyceride (TG) were determined using isopropanol, as described in Methods, and expressed as milligrams per gram of liver protein ( $n=4-5 ;{ }^{*} p<0.05,{ }^{* *} p<0.01$ vs. vehicle group); (e) mRNA expression of LDL receptor $(L D L R)$, scavenger receptor class $\mathrm{B}$ type I (SR-BI), $A B C A 1$, ATP-binding cassette subfamily G member 8 (ABCG8), PPAR $\gamma$, cholesterol 7- $\alpha$ hydroxylase (CYP7A1), cholesterol 27- $\alpha$ hydroxylase (CYP27A1), fatty acid synthase (FASN), PON1, IL-6, and TNF- $\alpha$ in livers of apoE ${ }^{-1-}$ mice was measured using qPCR with the expression level of each gene normalized to the level of $18 \mathrm{~S}$ RNA $(n=4-5$; * $p<0.05$, ** $p<0.01$ vs. vehicle group).

Liver is the central metabolic organ that plays a critical role in regulating lipid homeostasis, oxidative stress and inflammation. To investigate the underlying mechanisms of ISL on atherosclerosis and hepatic steatosis, we examined the effects of ISL on the hepatic expression of lipoprotein-metabolism-related genes in apoE $E^{-1-}$ mice. As shown in Figure 5e, the mRNA levels of scavenger receptor class $B$ type I (SR-BI), ABCA1, ATP-binding cassette subfamily G member 8 (ABCG8), PPAR $\gamma$, and the bile-acid-biosynthesis-related genes cholesterol 7- $\alpha$ hydroxylase (CYP7A1) and cholesterol 27- $\alpha$ hydroxylase (CYP27A1) were increased with statistical significance in the liver of apoE ${ }^{-/-}$mice fed with ISL compared with the vehicle group. The mRNA levels of a key enzyme in fatty acid synthesis 
(fatty acid synthase $(F A S N)$ ) was decreased, while LDL receptor (LDLR) expression levels were not changed. Furthermore, ISL significantly increased PON1 mRNA levels and decreased the mRNA levels of inflammatory factors $I L-6$ and TNF- $\alpha$ in the liver of apoE ${ }^{-/-}$mice (Figure 5e). These data suggested that ISL had the beneficial effects of enhancing hepatic lipid-selective uptake, inhibiting fatty acid synthesis, increasing cholesterol excretion into the bile, and improving the oxidative and inflammation status in the liver, which are involved in the amelioration of atherosclerosis and hepatic steatosis.

\section{Discussion}

It has been established that macrophages phagocytize oxidized lipids, transform into foam cells, and then deposit themselves in the arterial intima to form atherosclerotic plaques $[19,20]$. Reducing plasma lipid levels and the levels of inflammatory factors, and suppressing oxidative stress, may effectively inhibit the development of atherosclerotic plaques. The data presented here provide the first evidence that ISL attenuates the development of atherosclerosis and hepatic steatosis in Western diet-fed apoE $\mathrm{E}^{-/-}$mice, and the potential mechanism may include amelioration of lipid metabolism, inflammation, and oxidative status.

\subsection{ISL Inhibits the Inflammatory Responses In Vitro and In Vivo}

Chronic inflammation is a hallmark of atherosclerosis. Previous studies have indicated that the proinflammatory factors IL-6, TNF- $\alpha$, and the chemokine MCP- 1 are locally elevated in atherosclerotic plaques and promote macrophage recruitment to the atheromatous lesion [21,22]. Macrophages treated with LPS are induced to a proinflammatory phenotype and stimulate the expression of cytokines (e.g., TNF- $\alpha$, IL-6, IL-1 $\beta$ ) through the TLR4/Myd88/NFkB pathway [23]. Therefore, anti-inflammatory therapies that inhibit IL-6 and MCP-1 may be developed as novel antiatherosclerotic drugs [22,24]. The present study showed that ISL reduced LPS-stimulated macrophage inflammatory reactions by suppressing the mRNA expression levels of $I L-6, T N F-\alpha$, and $M C P-1$ in macrophages. In the in vivo study, plasma protein levels of IL-6, TNF- $\alpha$, and MCP-1 were also dramatically decreased in apoE ${ }^{-/-}$ mice fed ISL, and the mRNA levels of IL- 6 and TNF- $\alpha$ were decreased in the liver, indicating that ISL achieved its anti-inflammatory activities by inhibiting inflammatory cytokine production, and might possess the ability to attenuate atherosclerosis.

Cellular disbalance of cholesterol homeostasis is often accompanied by aggravated inflammation [25]. It has been reported that low-dose LPS potently disrupts cholesterol efflux from macrophages [26]. $\mathrm{LXR} \alpha$, a key regulator in lipid metabolism and transport and the transcription regulator of ABCA1, reportedly suppresses NFKB-mediated inflammatory signaling and is essential for maintaining macrophage homeostasis [27]. Our present study showed that the mRNA levels of $L X R \alpha$ and $A B C A 1$ were downregulated in peritoneal macrophages by LPS-induced inflammatory stress, which reduces cholesterol efflux from macrophages, consistent with those of recent reports [28,29]. We also found that ISL could increase the mRNA levels of $L X R \alpha$ and $A B C A 1$, contributing to facilitating the removal of excess lipids from macrophages. On the other hand, increased LXR $\alpha$ and ABCA1 levels might dampen proinflammatory signaling pathways. CD36 is the major receptor responsible for the uptake of ox-LDL [30]. Our in vitro data verified that ISL could reverse LPS-induced CD36 expression. Thus, we presume that the anti-inflammatory function of ISL is associated with maintaining the intracellular cholesterol homeostasis through the regulation of lipid transportation-related genes under inflammatory conditions.

\subsection{ISL Ameliorates Oxidative Stress In Vitro and In Vivo}

Growing evidence indicates that oxidative stress-which leads to dysfunctional molecules, inflammatory process, and cell damage-is integral in the development of atherosclerosis. Oxidative modification of LDL is really atherogenic compared with its native state. Oxidized LDL has several biological effects, including promoting foam cell formation, and proinflammatory, cytotoxic, immunogenicity, and other activities [1]. Ox-LDL has also been shown to upregulate vascular 
endothelial growth factor (VEGF) expression in macrophages as well as endothelial cells through activation of PPAR $\gamma$, which plays a major role in regulating lipid metabolism, inhibiting inflammation, and reducing oxidative stress [31]. Reportedly, ox-LDL acts as a PPAR $\gamma$ ligand; the activation of PPAR $\gamma$ promotes ox-LDL uptake by upregulating CD36 expression in monocytes and promotes cholesterol removal from macrophages through ABCA1 [32-34]. However, contrary to our expectation in our study, PPAR $\gamma$ expression level was downregulated by ox-LDL, and the protein levels of CD36 and ABCA1 were increased in peritoneal macrophages induced by ox-LDL. However, ISL treatment enhanced PPAR $\gamma$ expression levels, which was accompanied by decreased ABCA1 and CD36 protein levels. These results support that ISL could decrease oxysterol influx and the subsequent efflux of cellular cholesterol under stimulation of ox-LDL, indicating that the antioxidative role of ISL might benefit the inhibition of cholesterol accumulation in macrophages and foam cell formation. We presumed that this might be the result of different experimental conditions, such as the modified extent of ox-LDL and the treatment time. Chawla et al. also holds the view that PPAR $\gamma$ targets CD36 but is unnecessary for expression of this gene [35]. Therefore, whether through a direct or indirect relationship, ISL protects macrophages against oxidative damage and maintains cellular cholesterol homeostasis in macrophages, which might be related to the upregulation of the PPAR $\gamma$ signaling.

We further observed that ISL reduced atherosclerotic lesions in $\mathrm{apoE}^{-/-}$mice, and this action seemed to be associated with the antioxidant effect of ISL. Extracellular SOD is the main antioxidant enzyme that regulates circulating redox status [36]. In apoE $\mathrm{E}^{-/-}$mice, administration of ISL increases the activity of plasma SOD. PON1, another indicator of the antioxidant defense system in the circulation, is mainly synthesized in the liver, tightly located in HDL [37], and is responsible for most of the antioxidant properties of HDL [38]. We and others have demonstrated that lower plasma PON1 activity is associated with increased development and extent of atherosclerosis, and that high plasma PON1 activity has an antiatherosclerotic effect $[39,40]$. The present study showed that plasma PON1 activity and hepatic PON1 expression are increased in ISL-fed mice, providing an additional mechanistic link that ISL improves the antioxidative and anti-inflammatory function of plasma HDL, further contributing to protecting LDL/HDL against lipid peroxidation and reducing atherosclerosis.

\subsection{ISL Regulates Lipid Metabolism In Vivo}

Hyperlipidemia is the main cause of atherosclerosis. In our in vivo study, we found that ISL ameliorated plasma VLDL/LDL-C levels and hepatic lipid accumulation in Western diet-fed apoE ${ }^{-/-}$ mice. Because the liver is a central organ in the lipid metabolism-which involves lipid synthesis, apolipoprotein production, and lipid transport-and the final excretion of cholesterol from the body, we showed that ISL significantly upregulated the mRNA levels of hepatic cholesterol transporters SR-BI, ABCA1, and ABCG8, as well as CYP7A1 and CYP27A1, besides the role of antioxidation and anti-inflammation.

SR-BI, an HDL receptor abundantly expressed in the liver, is involved in selective hepatic uptake of spherical HDL cholesterol [41]. Researchers have demonstrated the critical role of hepatic SR-BI on reverse cholesterol transport (RCT) and its atheroprotective function in mouse models [42,43]. Therefore, our result showed that ISL increased the expression of SR-BI in hepatocytes, suggesting that ISL could assist HDL catabolism through promotion of hepatic selective cholesterol uptake.

$A B C$ superfamily proteins are critical membrane transporters that regulate the delivery and disposal of cholesterol [44]. Hepatic ABCA1 plays an essential role in nascent HDL formation by mediating the efflux of cell cholesterol and phospholipids to lipid-poor apolipoprotein AI (apoAI). ABCG8 plays an important role in the liver, specifically in the excretion of cholesterol into the bile [45]. Some studies have shown that ABCA1 and ABCG8 in the liver can prevent atherosclerosis by enhancing HDL biogenesis and hepatic cholesterol excretion [41]. Our present study suggests that ISL may increase cholesterol movement and promote HDL metabolism, including SR-BI-dependent HDL-C uptake, ABCG8-mediating secretion of cholesterol into the bile, and ABCA1-dependent secretion of nascent HDL, even without a net increase of plasma HDL-C levels. 
CYP7A1 and CYP27A1 are the key enzymes of bile-acid synthesis in liver. The data showing that their mRNA levels were significantly increased also supports that ISL enhanced the secretion of cholesterol into bile, a role that is consistent with the increase of ABCG8 mRNA levels. However, we did not directly measure fecal bile acids and cannot exclude nonhepatic mechanisms by which ISL may increase fecal cholesterol exit.

Besides the role on cholesterol metabolism, ISL was observed to decrease the hepatic mRNA levels of the lipogenic genes FASN in apoE ${ }^{-/-}$mice, indicating that ISL could reduce TG biosynthesis. We suggest that ISL performs regulation of lipogenesis and also contributes to diminished hepatic steatosis and attenuated atherosclerosis in Western-diet-fed apoE ${ }^{-/-}$mice.

PPAR $\gamma$ is one of the nuclear hormone-receptor superfamilies which induce hepatocyte peroxisome proliferation. The role of PPAR $\gamma$ activation in macrophage cholesterol homeostasis has been established, and studies suggest that PPAR $\gamma$ is involved in the development of atherosclerosis. The lipoprotein-metabolism-related genes—such as $S R-B I, A B C A 1$, and $A B C G 1$ - and the mRNA levels of inflammatory factors IL- 6 and TNF- $\alpha$ are all reported as targets of PPAR $\gamma$ [31]. Additionally, in our in vitro and in vivo studies, we found that ISL upregulates PPAR $\gamma$ expression in macrophages and liver. Therefore, regulation of PPAR $\gamma$ signaling might be an underlying mechanism that ISL enhanced, increasing the mRNA levels of SR-BI, ABCA1, ABCG8, and decreasing the mRNA levels of IL- 6 and $T N F-\alpha$ in the liver. These changes contribute to regulation of lipid homeostasis, reduction of oxidative stress, and inhibition of inflammation, mediated by PPAR $\gamma$.

\section{Materials and Methods}

\subsection{Materials and Reagents}

Dulbecco's Modified Eagle Medium (DMEM) was purchased from Thermo Fisher (Beijing, China). Fetal bovine serum (FBS) and antibiotics (streptomycin and penicillin) were obtained from GIBCO (New York, NY, USA). All tissue culture plasticware was obtained from Corning (Corning, NY, USA). ISL (purity: 98.26\%) was purchased from Shaanxi Green Bio-Engineering Co., Ltd. (Xi'an, China), and dissolved in $0.5 \%$ sodium carboxymethyl cellulose (CMC-Na). MTT, LPS, and oil red O were all from Sigma-Aldrich (St. Louis, MO, USA). LDL, $1 \mathrm{mg} / \mathrm{mL}$, was obtained from Prospect (Ness Ziona, Israel) and reacted with $10 \mu \mathrm{mol} / \mathrm{L}$ copper sulfate for $24 \mathrm{~h}$ at $37^{\circ} \mathrm{C}$. After dialysis, the LDL was filtered with a $0.22 \mu \mathrm{m}$ membrane resulting in ox-LDL; the concentration was determined using the Lowry method. Anti-CD36 and anti-ABCA1 monoclonal antibodies were obtained from Abcam (Cambridge, MA, USA). Anti-PPAR $\gamma$ polyclonal antibody and anti- $\beta$-actin monoclonal antibody were from Santa Cruz Biotechnology (Delaware Ave Santa Cruz, CA, USA). Horseradish peroxidase (HRP)-conjugated secondary antibodies were from Jackson Laboratory (Main St Bar Harbor, ME, USA). The enhanced chemiluminescence (ECL) kit was purchased from GE Healthcare (Waukesha, WI, USA). PCR primers were from Sangon Biotech (Shanghai, China). All other chemicals were of the best grade and obtained from commercial sources.

\subsection{Mice}

$\mathrm{ApoE}^{-/-}$mice of C57BL/6 background were purchased from Vital River Laboratory Animal Technology Company, China, and housed in microisolator cages under specific pathogen-free conditions at the Wuhan University Animal Center. Thirty female apoE ${ }^{-/-}$mice at 20 weeks of age were randomly divided into three groups and fed an American Institute of Nutrition (AIN76A) Western diet (HFK Bioscience Company, Beijing, China) supplemented with 0.5\% CMC-Na or ISL (either $20 \mathrm{mg} / \mathrm{kg} /$ day or $100 \mathrm{mg} / \mathrm{kg} /$ day) by intragastric gavage for 12-week. The animal care and experimental procedures were approved by the Medical Animal Care \& Welfare Committee (20140126056, 26 January 2014) for animal experiments of Wuhan University, and all experiments were performed according to the guidelines for the care and use of laboratory animals of the Chinese Animal Welfare Committee. 


\subsection{Cell Culture}

Peritoneal macrophages were collected from mice that were injected with $3 \mathrm{~mL} \mathrm{3 \%}$ thioglycolate $72 \mathrm{~h}$ prior to isolation. The macrophages were seeded in plates and incubated with serum-free DMEM at $37^{\circ} \mathrm{C}$ overnight before further treatment [46]. HeLa cells were obtained from the Animal Biosafety Level 3 Laboratory (ABSL-III) of the School of Basic Medicine, Wuhan University. The cells were cultured in DMEM containing 10\% fetal bovine serum and antibiotics $(100 \mathrm{mg} / \mathrm{mL}$ streptomycin, $100 \mathrm{U} / \mathrm{mL}$ penicillin; Beyotime, Haimen, Jiangsu, China) at $37^{\circ} \mathrm{C}$.

\subsection{Determination of Cytokine Expression Levels by Real-Time PCR}

Mouse peritoneal macrophages were cocultured with $50 \mathrm{ng} / \mathrm{mL}$ LPS or $50 \mu \mathrm{g} / \mathrm{mL}$ ox-LDL and $0.1 \mu \mathrm{g} / \mathrm{mL}$ ISL for $6 \mathrm{~h}$. The cells and fresh tissue sample were harvested using Trizol reagent (Invitrogen, Carlsbad City, CA, USA). Total RNA was extracted using an RNeasy kit (Qiagen, Valencia, CA, USA) and reverse transcribed into cDNA using an iScript cDNA Synthesis kit (Bio-Rad, Hercules, CA, USA). Real-time qPCR measurements of target mRNA levels were performed on an Eppendorf Realplex2 Mastercycler (Eppendorf, Hamburg, Germany) according to the manufacturer's instructions. Mouse genes were normalized with $18 \mathrm{~S}$ rRNA as endogenous controls. The PCR primers for amplification of mouse genes, $18 S, I L-6, T N F-\alpha$, and $M C P-1$ are shown in Table S1.

\subsection{Determination of Protein Expression by Western Blot}

Harvested cells and frozen tissue samples were lysed using radioimmunoprecipitation assay (RIPA) (Beyotime Institute of Biotechnology, Haimen, Jiangsu, China) with 1\% proteinase inhibitors (Roche, Basel, Switzerland). The supernatant was determined using the Lowry method with a DC protein assay kit (Bio-Rad, Alfred Nobel Drive Hercules, CA, USA), and appropriate amounts of proteins were loaded and separated using sodium dodecyl sulfate polyacrylamide gel electrophoresis (SDS-PAGE); Western blotting was performed as previously described [47]. The primary antibodies included monoclonal CD36 (1:1000), monoclonal ABCA1 (1:1000), polyclonal PPAR $\gamma$ (1:500), monoclonal $\beta$-actin (1:1000), and HRP-conjugated secondary antibody $(1: 10,000)$. Signals were detected using an enhanced chemiluminescence kit (ECL, GE Healthcare, Waukesha, WI, USA). The band intensity was quantitated using densitometry on Image J software (NIH, Bethesda, MD, USA).

\subsection{Plasma Analysis}

Plasma was collected by retro-orbital venous plexus puncture, and immediately separated by centrifugation at $10,000 \times g$ for $10 \mathrm{~min}$ at $4{ }^{\circ} \mathrm{C}$. Plasma TC and TG levels were measured using enzymatic colorimetric assays (Mind Bioengineering, Shanghai, China). Plasma lipoprotein profiles were determined by FPLC using a Superose 6 10/300 GL column (Amersham Biosciences, Waukesha, WI, USA) on an AKTA purifier (GE Healthcare, Waukesha, WI, USA).

Plasma IL-6, TNF- $\alpha$, and MCP-1 levels were determined using enzyme-linked immunosorbent assay (ELISA) kits (eBioscience, San Diego, CA, USA) according to the manufacturer's instructions. The activity of plasma SOD was determined using enzymatic colorimetric assay on SOD reagent kits (Nanjing Jiancheng Bioengineering Institute, Nanjing, China). Plasma PON1 activity was measured using paraoxon (Sigma-Aldrich, St. Louis, MO, USA) as substrate, as previously described [40].

\subsection{Atherosclerosis Lesion Analysis}

After supplementation with ISL for 12-week, the mice were sacrificed. For en face analysis, the aortas were dissected and fixed in $4 \%$ paraformaldehyde at room temperature overnight. The fatty tissue was removed, and the samples were stained with oil red $O$. The plaque areas were analyzed using Image J software. The aortic roots were embedded in optimum cutting temperature compound (OCT) (SAKURA, Torranc, CA, USA) and fast frozen at $-20{ }^{\circ} \mathrm{C}$. The aortic sinuses were cut into $8 \mu \mathrm{m}$ 
serial sections for further analysis. The extent of atherosclerosis was determined by oil red $\mathrm{O}$ staining and quantified using Image J software, as previously described [48].

\title{
4.8. Gene Expression, Biochemical, and Histochemical Analyses of the Liver
}

At the endpoint, mouse liver tissues were homogenized using Trizol reagent. Gene expression was determined as described in Section 4.4. The PCR primers for amplification of mouse genes LDLR, SR-BI, $L X R \alpha, A B C A 1, A B C G 8, P P A R \gamma, C Y P 7 A 1, C Y P 27 A 1, F A S N$, and PON1 are shown in Supplementary Table S1. Fresh liver tissue was homogenized in phosphate-buffered saline (PBS), and total lipids were extracted using isopropanol. The amounts of TC, FC, and TG were determined as previously described [48]. Lipid content was normalized to liver protein content. The liver tissue was fixed in $4 \%$ paraformaldehyde at room temperature overnight, and performed the histochemical analysis using HE staining. The fresh liver tissue was embedded in OCT and cut into $5 \mu \mathrm{m}$ serial sections for oil red $\mathrm{O}$ staining, and images were acquired using an Olympus microscope.

\subsection{Statistical Analysis}

The data given represent the mean of 3 experiments \pm standard error of the mean for in vitro experiment. Statistical analyses were performed using GraphPad Prism software 5.0 (GraphPad Software, Inc., La Jolla, CA, USA). Statistical significance was evaluated using an one-way analysis of variance (ANOVA) followed by a post-hoc Newman-Keuls Multiple Comparison Test. Differences were considered to be significant at $p<0.05$.

\section{Conclusions}

In summary, our present study shows that ISL, a bioactive ingredient of flavonoids, reduces plasma VLDL/LDL, improves HDL function and plasma redox status, and suppresses macrophage and liver inflammation, resulting in reduced atherosclerotic plaque development and diminished hepatic steatosis in Western diet-fed apoE $\mathrm{E}^{-/-}$mice. Our results suggest that ISL might be developed as a new antiatherosclerosis therapy. However, the specific mechanism of ISL, meaning the precise molecular targets through which ISL achieves the above functions, warrants further investigation.

Supplementary Materials: Supplementary materials can be found at www.mdpi.com/1422-0067/17/11/1932/s1.

Acknowledgments: This work was supported by grants from the National Natural Science Foundation of China ( 81072559 to Fen Du, 81270364 to Hong Yu and 81570417 to Dongfang Wu), in part by the grant from the Natural Science Foundation of Hubei Province, China (2014CFB143) and the Health and Family Planning Commission of Wuhan Municipality, China (WRJK0901). We thank Daping Fan at the University of South Carolina for proofreading the manuscript and his invaluable advice.

Author Contributions: Fen Du analyzed the data, wrote the paper; Quzhen Gesang performed the experiments; Jia Cao contributed to Western blot; Mei Qian carried out q-PCR; Li Ma participated in animal experiments; Dongfang Wu participated in design the experiments; Hong Yu conceived and designed the experiments.

Conflicts of Interest: The authors declare no conflict of interest. The founding sponsors had no role in the design of the study; in the collection, analyses, or interpretation of data; in the writing of the manuscript, and in the decision to publish the results.

\author{
Abbreviations \\ ABCA1 ATP-binding cassette transporter A1 \\ apoE $^{-/-} \quad$ apolipoprotein E deficient \\ FPLC fast protein liquid chromatography \\ HDL high density lipoprotein \\ HE hematoxylin and eosin \\ HRP horseradish peroxidase \\ IL Interleukin \\ ISL isoliquiritigenin \\ LDL low density lipoproteins \\ LPS lipopolysaccharide
}




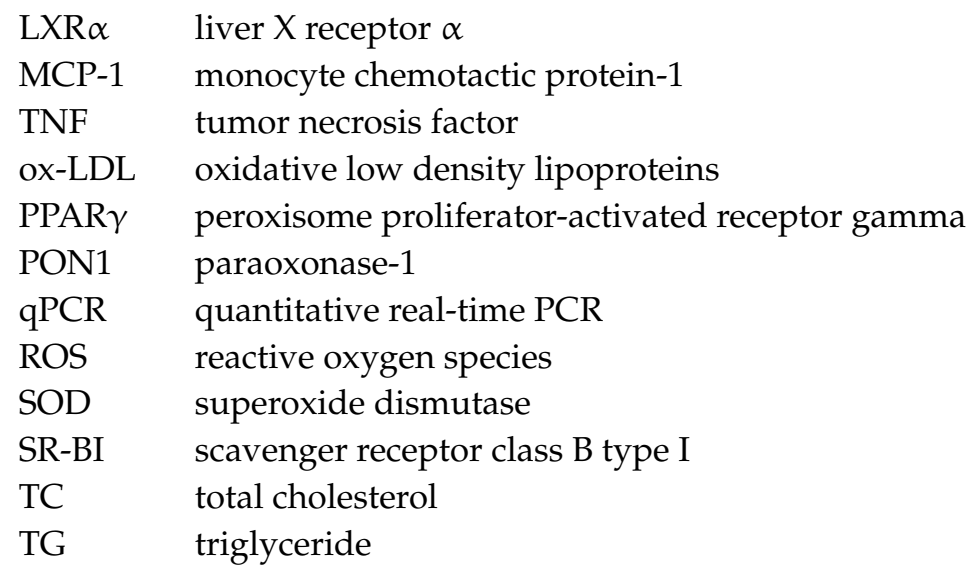

\section{References}

1. Madamanchi, N.R.; Vendrov, A.; Runge, M.S. Oxidative stress and vascular disease. Arterioscler. Thromb. Vasc. Biol. 2005, 25, 29-38. [CrossRef] [PubMed]

2. Steinberg, D. Atherogenesis in perspective: Hypercholesterolemia and inflammation as partners in crime. Nat. Med. 2002, 8, 1211-1217. [CrossRef] [PubMed]

3. Mizuno, Y.; Jacob, R.F.; Mason, R.P. Inflammation and the development of atherosclerosis. J. Atheroscler. Thromb. 2011, 18, 351-358. [CrossRef] [PubMed]

4. Tedgui, A.; Mallat, Z. Cytokines in atherosclerosis: Pathogenic and regulatory pathways. Physiol. Rev. 2006, 86, 515-581. [CrossRef] [PubMed]

5. Hansson, G.K. Inflammation, atherosclerosis, and coronary artery disease. N. Engl. J. Med. 2005, 352, $1685-1695$. [CrossRef] [PubMed]

6. Zampetaki, A.; Dudek, K.; Mayr, M. Oxidative stress in atherosclerosis: The role of micrornas in arterial remodeling. Free Radic. Biol. Med. 2013, 64, 69-77. [CrossRef] [PubMed]

7. Tiwari, R.L.; Singh, V.; Barthwal, M.K. Macrophages: An elusive yet emerging therapeutic target of atherosclerosis. Med. Res. Rev. 2008, 28, 483-544. [CrossRef] [PubMed]

8. Weber, C.; Noels, H. Atherosclerosis: Current pathogenesis and therapeutic options. Nat. Med. 2011, 17, 1410-1422. [CrossRef] [PubMed]

9. Feng Yeh, C.; Wang, K.C.; Chiang, L.C.; Shieh, D.E.; Yen, M.H.; San Chang, J. Water extract of licorice had anti-viral activity against human respiratory syncytial virus in human respiratory tract cell lines. J. Ethnopharmacol. 2013, 148, 466-473. [CrossRef] [PubMed]

10. Peng, F.; Du, Q.; Peng, C.; Wang, N.; Tang, H.; Xie, X.; Shen, J.; Chen, J. A review: The pharmacology of isoliquiritigenin. Phytother. Res. 2015, 29, 969-977. [CrossRef] [PubMed]

11. Zhao, Z.; Park, S.M.; Guan, L.; Wu, Y.; Lee, J.R.; Kim, S.C.; Kim, Y.W.; Zhao, R. Isoliquiritigenin attenuates oxidative hepatic damage induced by carbon tetrachloride with or without buthionine sulfoximine. Chem. Biol. Interact. 2015, 225, 13-20. [CrossRef] [PubMed]

12. Honda, H.; Nagai, Y.; Matsunaga, T.; Okamoto, N.; Watanabe, Y.; Tsuneyama, K.; Hayashi, H.; Fujii, I.; Ikutani, M.; Hirai, Y.; et al. Isoliquiritigenin is a potent inhibitor of NLRP3 inflammasome activation and diet-induced adipose tissue inflammation. J. Leukoc. Biol. 2014, 96, 1087-1100. [CrossRef] [PubMed]

13. Watanabe, Y.; Nagai, Y.; Honda, H.; Okamoto, N.; Yamamoto, S.; Hamashima, T.; Ishii, Y.; Tanaka, M.; Suganami, T.; Sasahara, M.; et al. Isoliquiritigenin attenuates adipose tissue inflammation in vitro and adipose tissue fibrosis through inhibition of innate immune responses in mice. Sci. Rep. 2016, 6, 23097-23113. [CrossRef] [PubMed]

14. Wu, S.; Xue, J.; Yang, Y.; Zhu, H.; Chen, F.; Wang, J.; Lou, G.; Liu, Y.; Shi, Y.; Yu, Y.; et al. Isoliquiritigenin inhibits interferon- $\gamma$-inducible genes expression in hepatocytes through down-regulating activation of JAK1/STAT1, IRF3/MyD88, ERK/MAPK, JNK/MAPK and PI3K/Akt signaling pathways. Cell. Physiol. Biochem. 2015, 37, 501-514. [CrossRef] [PubMed]

15. Park, S.J.; Choe, Y.G.; Kim, J.H.; Chang, K.T.; Lee, H.S.; Lee, D.S. Isoliquiritigenin impairs insulin signaling and adipocyte differentiation through the inhibition of protein-tyrosine phosphatase 1B oxidation in 3T3-L1 preadipocytes. Food Chem. Toxicol. 2016, 93, 5-12. [CrossRef] [PubMed] 
16. Cao, L.-J.; Li, H.-D.; Yan, M.; Li, Z.-H.; Gong, H.; Jiang, P.; Deng, Y.; Fang, P.-F.; Zhang, B.-K. The protective effects of isoliquiritigenin and glycyrrhetinic acid against triptolide-induced oxidative stress in HepG2 cells involve Nrf2 activation. Evid.-Based Complement. Altern. Med. 2016, 2016, 1-8. [CrossRef] [PubMed]

17. Yang, E.J.; Min, J.S.; Ku, H.Y.; Choi, H.S.; Park, M.K.; Kim, M.K.; Song, K.S.; Lee, D.S. Isoliquiritigenin isolated from glycyrrhiza uralensis protects neuronal cells against glutamate-induced mitochondrial dysfunction. Biochem. Biophys. Res. Commun. 2012, 421, 658-664. [CrossRef] [PubMed]

18. Kim, Y.M.; Kim, T.H.; Kim, Y.W.; Yang, Y.M.; Ryu, D.H.; Hwang, S.J.; Lee, J.R.; Kim, S.C.; Kim, S.G. Inhibition of liver $\mathrm{X}$ receptor- $\alpha$-dependent hepatic steatosis by isoliquiritigenin, a licorice antioxidant flavonoid, as mediated by JNK1 inhibition. Free Radic. Biol. Med. 2010, 49, 1722-1734. [CrossRef] [PubMed]

19. Webb, N.R.; Moore, K.J. Macrophage-derived foam cells in atherosclerosis: Lessons from murine models and implications for therapy. Curr. Drug Targets 2007, 8, 1249-1263. [CrossRef] [PubMed]

20. Vittone, F.; Liberman, A.; Vasic, D.; Ostertag, R.; Esser, M.; Walcher, D.; Ludwig, A.; Marx, N.; Burgmaier, M. Sitagliptin reduces plaque macrophage content and stabilises arteriosclerotic lesions in $A p o e^{-/-}$mice. Diabetologia 2012, 55, 2267-2275. [CrossRef] [PubMed]

21. Qu, D.; Liu, J.; Lau, C.W.; Huang, Y. IL-6 in diabetes and cardiovascular complications. Br. J. Pharmacol. 2014, 171, 3595-3603. [CrossRef] [PubMed]

22. Lin, J.; Kakkar, V.; Lu, X. Impact of MCP-1 in atherosclerosis. Curr. Pharm. Des. 2014, 20, 4580-4588. [CrossRef] [PubMed]

23. Madonna, R.; de Caterina, R. Relevance of new drug discovery to reduce NF- $\mathrm{B}$ activation in cardiovascular disease. Vascul. Pharmacol. 2012, 57, 41-47. [CrossRef] [PubMed]

24. Ridker, P.M.; Luscher, T.F. Anti-inflammatory therapies for cardiovascular disease. Eur. Heart J. 2014, 35, 1782-1791. [CrossRef] [PubMed]

25. Tietge, U.J. Hyperlipidemia and cardiovascular disease: Inflammation, dyslipidemia, and atherosclerosis. Curr. Opin. Lipidol. 2014, 25, 94-95. [CrossRef] [PubMed]

26. Maitra, U.; Li, L. Molecular mechanisms responsible for the reduced expression of cholesterol transporters from macrophages by low-dose endotoxin. Arterioscler. Thromb. Vasc. Biol. 2013, 33, 24-33. [CrossRef] [PubMed]

27. Im, S.S.; Osborne, T.F. Liver X receptors in atherosclerosis and inflammation. Circ. Res. 2011, 108, 996-1001. [CrossRef] [PubMed]

28. Ma, K.L.; Ruan, X.Z.; Powis, S.H.; Chen, Y.; Moorhead, J.F.; Varghese, Z. Inflammatory stress exacerbates lipid accumulation in hepatic cells and fatty livers of apolipoprotein e knockout mice. Hepatology 2008, 48, 770-781. [CrossRef] [PubMed]

29. Wang, Y.; Moser, A.H.; Shigenaga, J.K.; Grunfeld, C.; Feingold, K.R. Downregulation of liver X receptor- $\alpha$ in mouse kidney and HK-2 proximal tubular cells by lps and cytokines. J. Lipid Res. 2005, 46, 2377-2387. [CrossRef] [PubMed]

30. Kunjathoor, V.V.; Febbraio, M.; Podrez, E.A.; Moore, K.J.; Andersson, L.; Koehn, S.; Rhee, J.S.; Silverstein, R.; Hoff, H.F.; Freeman, M.W. Scavenger receptors class A-I/II and CD36 are the principal receptors responsible for the uptake of modified low density lipoprotein leading to lipid loading in macrophages. J. Biol. Chem. 2002, 277, 49982-49988. [CrossRef] [PubMed]

31. Hamblin, M.; Chang, L.; Fan, Y.; Zhang, J.; Chen, Y.E. PPARs and the cardiovascular system. Antioxid. Redox Signal. 2009, 11, 1415-1452. [CrossRef] [PubMed]

32. Nagy, L.; Tontonoz, P.; Alvarez, J.G.; Chen, H.; Evans, R.M. Oxidized LDL regulates macrophage gene expression through ligand activation of PPAR $\gamma$. Cell 1998, 93, 229-240. [CrossRef]

33. Tontonoz, P.; Nagy, L.; Alvarez, J.G.; Thomazy, V.A.; Evans, R.M. PPAR $\gamma$ promotes monocyte/macrophage differentiation and uptake of oxidized LDL. Cell 1998, 93, 241-252. [CrossRef]

34. Chawla, A.; Boisvert, W.A.; Lee, C.H.; Laffitte, B.A.; Barak, Y.; Joseph, S.B.; Liao, D.; Nagy, L.; Edwards, P.A.; Curtiss, L.K.; et al. A PPAR $\gamma$-LXR-ABCA1 pathway in macrophages is involved in cholesterol efflux and atherogenesis. Mol. Cell 2001, 7, 161-171. [CrossRef]

35. Chawla, A.; Barak, Y.; Nagy, L.; Liao, D.; Tontonoz, P.; Evans, R.M. PPAR $\gamma$ dependent and independent effects on macrophage-gene expression in lipid metabolism and inflammation. Nat. Med. 2001, 7, 48-52. [PubMed]

36. Abreu, I.A.; Cabelli, D.E. Superoxide dismutases-A review of the metal-Associated mechanistic variations. Biochim. Biophys. Acta 2010, 1804, 263-274. [CrossRef] [PubMed] 
37. Vakili, L.; Hama, S.; Kim, J.B.; Tien, D.; Safarpoor, S.; Ly, N.; Vakili, G.; Hough, G.; Navab, M. The effect of HDL mimetic peptide 4F on PON1. Adv. Exp. Med. Biol. 2010, 660, 167-172. [PubMed]

38. Litvinov, D.; Mahini, H.; Garelnabi, M. Antioxidant and anti-inflammatory role of paraoxonase 1: Implication in arteriosclerosis diseases. N. Am. J. Med. Sci. 2012, 4, 523-532. [PubMed]

39. Mackness, B.; Mackness, M. Anti-inflammatory properties of paraoxonase-1 in atherosclerosis. Adv. Exp. Med. Biol. 2010, 660, 143-151. [PubMed]

40. Zhou, C.; Cao, J.; Shang, L.; Tong, C.; Hu, H.; Wang, H.; Fan, D.; Yu, H. Reduced paraoxonase 1 activity as a marker for severe coronary artery disease. Dis. Markers 2013, 35, 97-103. [CrossRef] [PubMed]

41. Fitzgerald, M.L.; Mujawar, Z.; Tamehiro, N. ABC transporters, atherosclerosis and inflammation. Atherosclerosis 2010, 211, 361-370. [CrossRef] [PubMed]

42. Trigatti, B.L.; Krieger, M.; Rigotti, A. Influence of the HDL receptor SR-BI on lipoprotein metabolism and atherosclerosis. Arterioscler. Thromb. Vasc. Biol. 2003, 23, 1732-1738. [CrossRef] [PubMed]

43. Mineo, C.; Shaul, P.W. Functions of scavenger receptor class B, type I in atherosclerosis. Curr. Opin. Lipidol. 2012, 23, 487-493. [CrossRef] [PubMed]

44. Oram, J.F.; Vaughan, A.M. ATP-binding cassette cholesterol transporters and cardiovascular disease. Circ. Res. 2006, 99, 1031-1043. [CrossRef] [PubMed]

45. Graf, G.A.; Yu, L.; Li, W.P.; Gerard, R.; Tuma, P.L.; Cohen, J.C.; Hobbs, H.H. ABCG5 and ABCG8 are obligate heterodimers for protein trafficking and biliary cholesterol excretion. J. Biol. Chem. 2003, 278, 48275-48282. [CrossRef] [PubMed]

46. Du, F.; Yu, F.; Wang, Y.; Hui, Y.; Carnevale, K.; Fu, M.; Lu, H.; Fan, D. MicroRNA-155 deficiency results in decreased macrophage inflammation and attenuated atherogenesis in apolipoprotein E-deficient mice. Arterioscler. Thromb. Vasc. Biol. 2014, 34, 759-767. [CrossRef] [PubMed]

47. Xu, Y.Y.; Du, F.; Meng, B.; Xie, G.H.; Cao, J.; Fan, D.; Yu, H. Hepatic overexpression of methionine sulfoxide reductase a reduces atherosclerosis in apolipoprotein E-deficient mice. J. Lipid Res. 2015, 56, 1891-1900. [CrossRef] [PubMed]

48. Yu, H.; Zhang, W.; Yancey, P.G.; Koury, M.J.; Zhang, Y.; Fazio, S.; Linton, M.F. Macrophage apolipoprotein E reduces atherosclerosis and prevents premature death in apolipoprotein $\mathrm{E}$ and scavenger receptor-class BI double-knockout mice. Arterioscler. Thromb. Vasc. Biol. 2006, 26, 150-156. [CrossRef] [PubMed] 